\title{
Retroneumoperitoneo secundario a cistitis enfisematosa
}

\section{Retropneumoperitoneum secondary to emphysematous}

cystitis

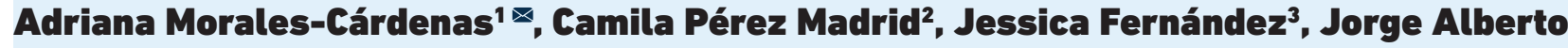 Carrillo-Bayona"}

1. Médica, epidemióloga, residente de radiología Universidad del Rosario.

2. Médica, epidemióloga, residente de dermatología, Universidad CES

3. Médico interno. Universidad del Rosario, Hospital Universitario Mayor Méderi, Bogotá, Colombia.

4. Médico Radiólogo. Hospital Universitario Mayor Méderi, Bogotá, Colombia.

Este trabajo cumple con las siguientes características editoriales

Fecha correspondencia: Recibido: noviembre 13 de 2015. Revisado: marzo 14 de 2016. Aceptado: abril 12 de 2016.

Forma de citar: Morales-Cárdenas A, PérezMadrid C, Fernández J, CarrilloBayona JA. Retroneumoperitoneo secundario a cistitis enfisematosa. Rev CES Med 2016 30(1): 99-106.

Open access

(C) Derecho de autor Licencia creative commons

Etica de publicaciones

Revisión por pares

Gestión por Open Journal System

ISSN 0129-8705

ISSN-e 2215-9177

Comparte

\section{CDS MEDICINA}

\section{Resumen}

La cistitis enfisematosa se define como la presencia de gas en la pared vesical secundaria a infección por microorganismos productores de gas. Es más frecuente en el género femenino, con predominio entre la sexta y octava década de la vida. Los factores de riesgo identificados son inmunosupresión, alteraciones estructurales o neurológicas del tracto urinario inferior, entre otros. Su prevalencia o incidencia es desconocida, dada la baja frecuencia de la enfermedad. Su forma de presentación clínica es muy variable e incluye desde un paciente asintomático hasta sepsis fulminante. El diagnóstico se puede realizar a través de estudios complementarios por imágenes o por métodos de visualización directa como la cistoscopia, laparotomía o laparoscopia, requiriendo rescate bacteriológico para confirmar la etiología infecciosa, siendo el método diagnóstico más usado la tomografía computarizada.

Presentamos el caso de una paciente femenina con inmunosupresión crónica por esteroides a altas dosis como parte de manejo de lupus eritematoso sistémico retroneumoperitoneo y enfisema de tejidos blandos pélvicos secundario a cistitis enfisematosa por Klebsiella pneaumoniae. Se trata del primer caso reportado de retroneumoperitoneo secundario a cistitis enfisematosa en una paciente con lupus eritematoso sistémico cuyo único factor de riesgo identificado fue la inmunosupresión farmacológica con esteroides.

Palabras clave: Cistitis enfisematosa, Infección del tracto urinario, Tomografía computada multidetector, Lupus eritematoso sistémico, Retroneumoperitoneo.

\footnotetext{
Abstract

Emphysematous cystitis is defined as the presence of gas in the bladder wall due to infection by gas-forming organisms. It is more common in females, predominantly between the sixth and eighth decades of life. The identified risk factors are immunosuppression (secondary to systemic diseases or drugs); structural or neurological lower urinary tract alterations,
} 
Enero - Junio 2016 - Pág 100

El diagnóstico se realiza a través de estudios complementarios por imágenes o visualización directa (cistoscopia, laparotomía o autopsia). radioscopiafuncionales respiratorias y de imagen, como la radiografía, la tomografía axial computadorizada tóraco-abdominal o la radioscopia. among others. Prevalence or incidence is unknown. Clinical manifestations are variable, ranges from an asymptomatic patient to fulminant sepsis. Although diagnosis can be made through complementary diagnostic imaging or direct visualization methods such as cystoscopy, laparotomy or laparoscopy, requiring bacteriological rescue to confirm infectious etiology. The most frequently diagnostic tool used is computed tomography. We report the case of a patient with systemic lupus erythematosus presenting with retropneumoperitoneum secondary to emphysematous cystitis due to infection by Klebsiella pneumonia.

This is the first reported case of retropneumoperitoneum secundary to emphysematous cystitis in a patient with systemic lupus erythematosus whose only risk factor identified was the pharmacological immunosuppression with steroids.

Keywords: Emphysematous cystitis, Urinary tract infection, High resolution computed tomography, Systemic lupus erythematosus, Retropneumoperitoneum.

\section{Introducción}

La cistitis enfisematosa es una complicación poco frecuente de las infecciones del tracto urinario que se caracteriza por la presencia de aire en la pared o lumen vesical, debido a la colonización por microrganismos formadores de gas, siendo los más frecuentes Escherichia coli y Klebsiella pneumoniae (1,2).

Se describe en algunos casos extensión del aire al espacio retroperitoneal, con aumento de la morbimortalidad. En los casos reportados, el factor de riesgo determinante es el antecedente de vejiga neurogénica en pacientes diabéticos, dada la alteración estructural e inmunosupresión $(1,2)$. La manifestación clínica más frecuente es dolor abdominal asociado a síntomas irritativos urinarios y el más específico la neumaturia, aunque su espectro clínico es amplio.

El diagnóstico se realiza a través de estudios complementarios por imágenes o visualización directa (cistoscopia, laparotomía o autopsia). En las imágenes diagnósticas se incluye la radiografía simple de abdomen en la que se identifica un halo radiolúcido que delimita la pared vesical con aire, en el área de proyección de la vejiga. La tomografía computarizada tiene mayor sensibilidad y especificidad en la caracterización del proceso enfisematoso a nivel vesical como así también su extensión extravesical, siendo este último uno de los principales factores que determinan la morbimortalidad del paciente.

Presentamos el caso de una paciente con retroneumoperitoneo secundario a cistitis enfisematosa, cuyo factor predisponente fue el antecedente de lupus eritematoso sistémico con inmunosupresión secundaria a altas dosis de esteroide.

\section{Reporte del caso}

Se trata de una paciente femenina de 50 años con antecedente de lupus eritematoso sistémico en tratamiento desde hace un año con prednisona $(50 \mathrm{mg} / \mathrm{día})$, cloroquina (150 mg/día) y micofenolato mofetil ( $2 \mathrm{~g} / \mathrm{día}$ ). Consultó por un cuadro clínico de tres semanas de hiporexia, intolerancia a la vía oral, deposiciones líquidas sin moco ni sangre y pérdida de $12 \mathrm{~kg}$ en un mes. Se evidenció pancitopenia en el hemograma y el urocultivo reportó infección de vías urinarias con aislamiento de Klebsiella pneumoniae multisensible.

Debido a la enfermedad diarréica crónica se le realizó colonoscopia, en la cual se encontró pancolitis, así como una tomografía computarizada de abdomen en la que 
se evidenció aire en la pared vesical que se extendía a los tejidos blandos pélvicos y al retroperitoneo, compatible con cistitis enfisematosa (figuras 1 y 2).

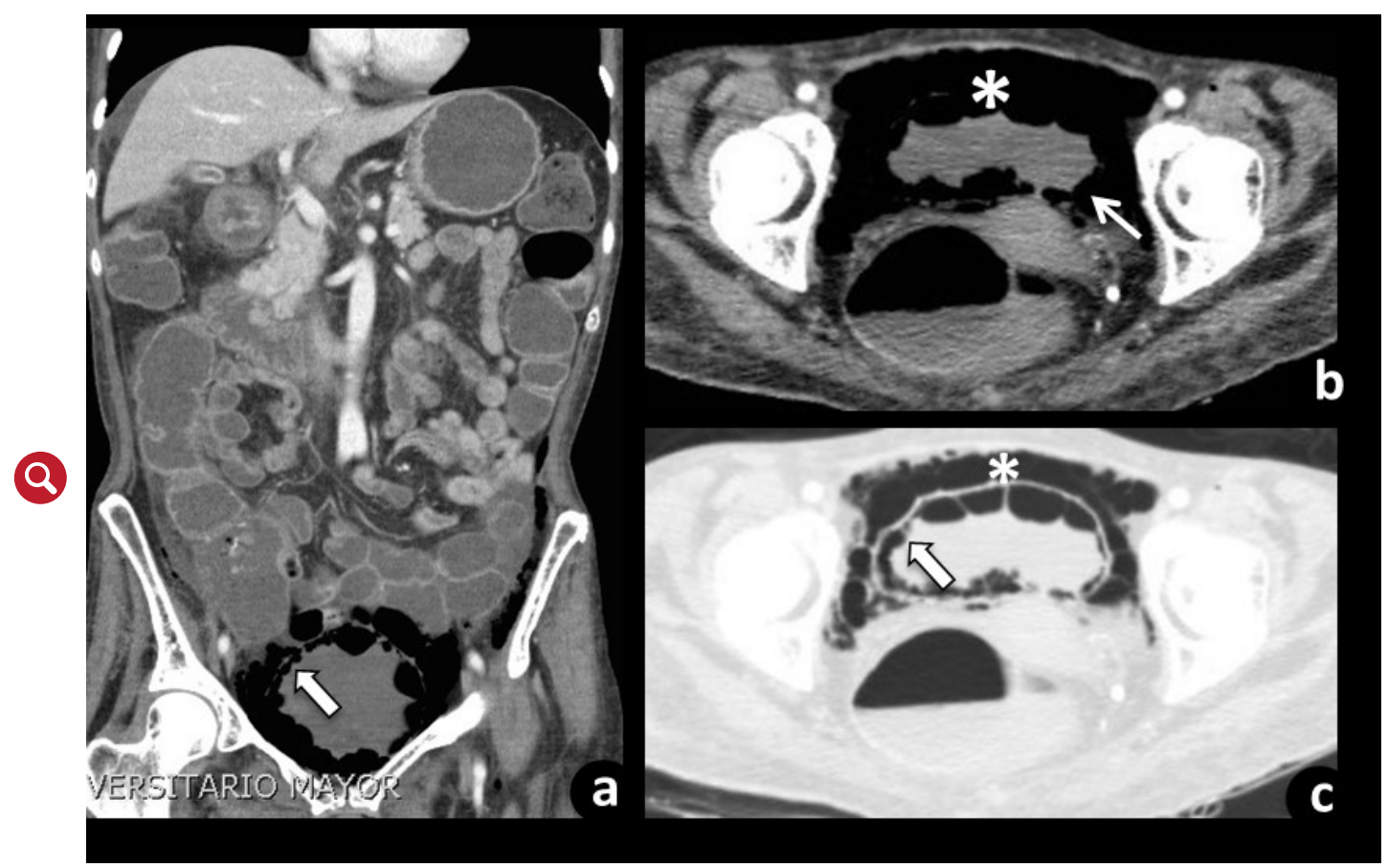

Figura 1. Tomografía abdominal contrastada, cortes axiales y reconstrucción coronal. En la imagen se observa aire en la pared vesical (flecha) (a y c) y en el espacio prevesical (asterisco) (b y c).

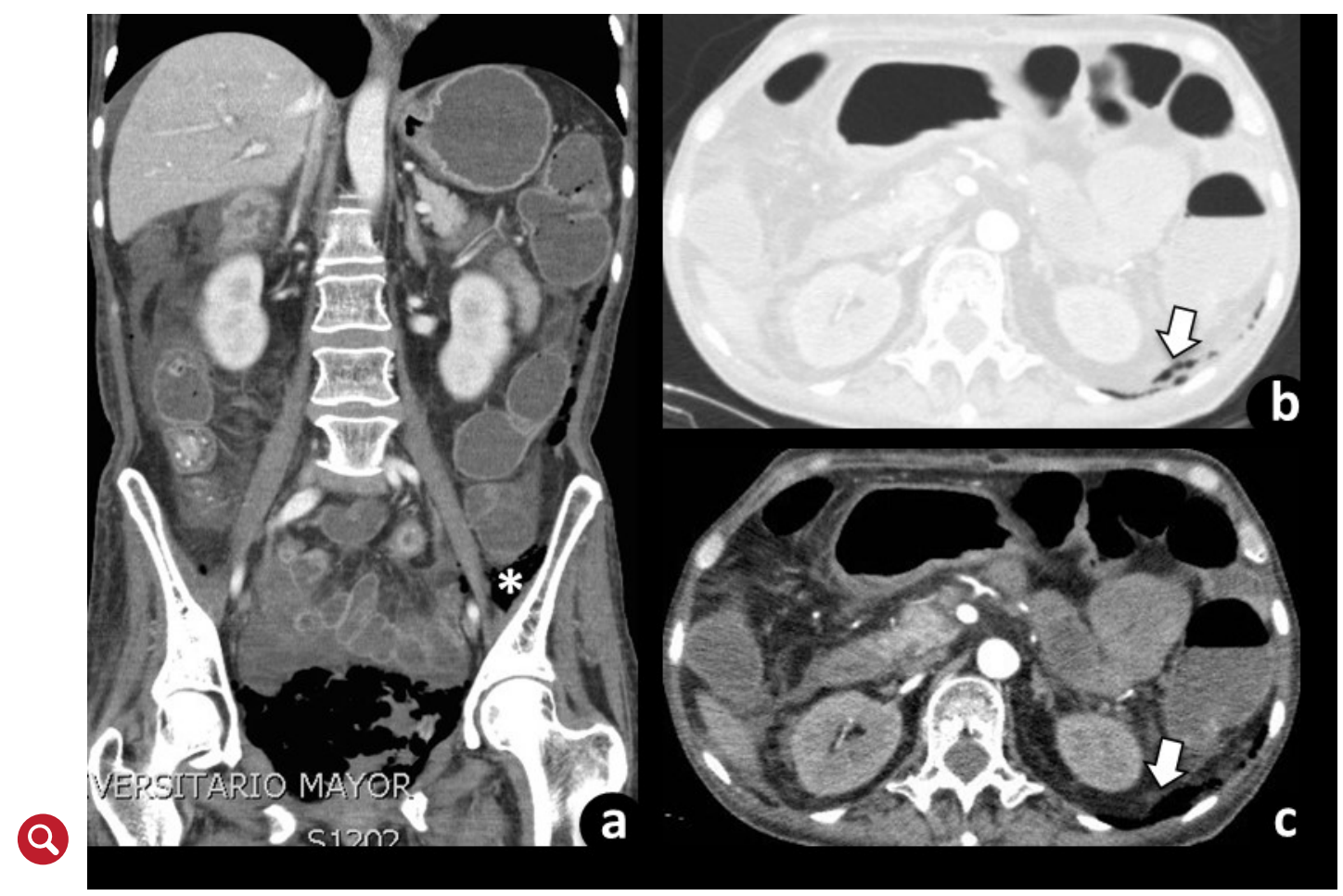

Figura 2. Tomografía abdominal contrastada, cortes axiales y reconstrucción coronal. En la imagen se observa aire en el espacio retroperitoneal adyacente al plano del músculo psoas iliaco izquierdo (asterisco) (a) y en el espacio pararrenal posterior (flecha) (b y c). 
Enero - Junio 2016 - Pág 102

Se trata de una complicación rara de las infecciones del tracto urinario, por lo que se desconocen datos de su prevalencia e incidencia. La cistitis enfisematosa es más frecuente en el género femenino y un pico de incidencia entre la sexta y octava década de la vida.
Se inició manejo antibiótico con ampicilina/sulbactam (3 g c/6h) y derivación urinaria permanente con sonda uretral. La evolución inicial fue tórpida, evidenciada por cifras tensionales en el límite inferior, taquicardia, coagulación intravascular diseminada (aparición de lesiones en piel con alteración en los tiempos de coagulación), progresión de la pancitopenia y signos de hipoperfusión (gases arteriales con tasa de extracción de oxígeno > 30 \%) compatible con sepsis severa.

Por lo anterior es trasladada a la unidad de cuidados intensivos, en donde se le inició manejo con milrinone y se adicionó vancomicina al manejo antibiótico. Se realizó nueva tomografía abdominal simple de control a los 10 días pos-tratamiento que evidenció disminución del neumoretroperitoneo y del enfisema de tejidos blandos pélvico (figura 3).

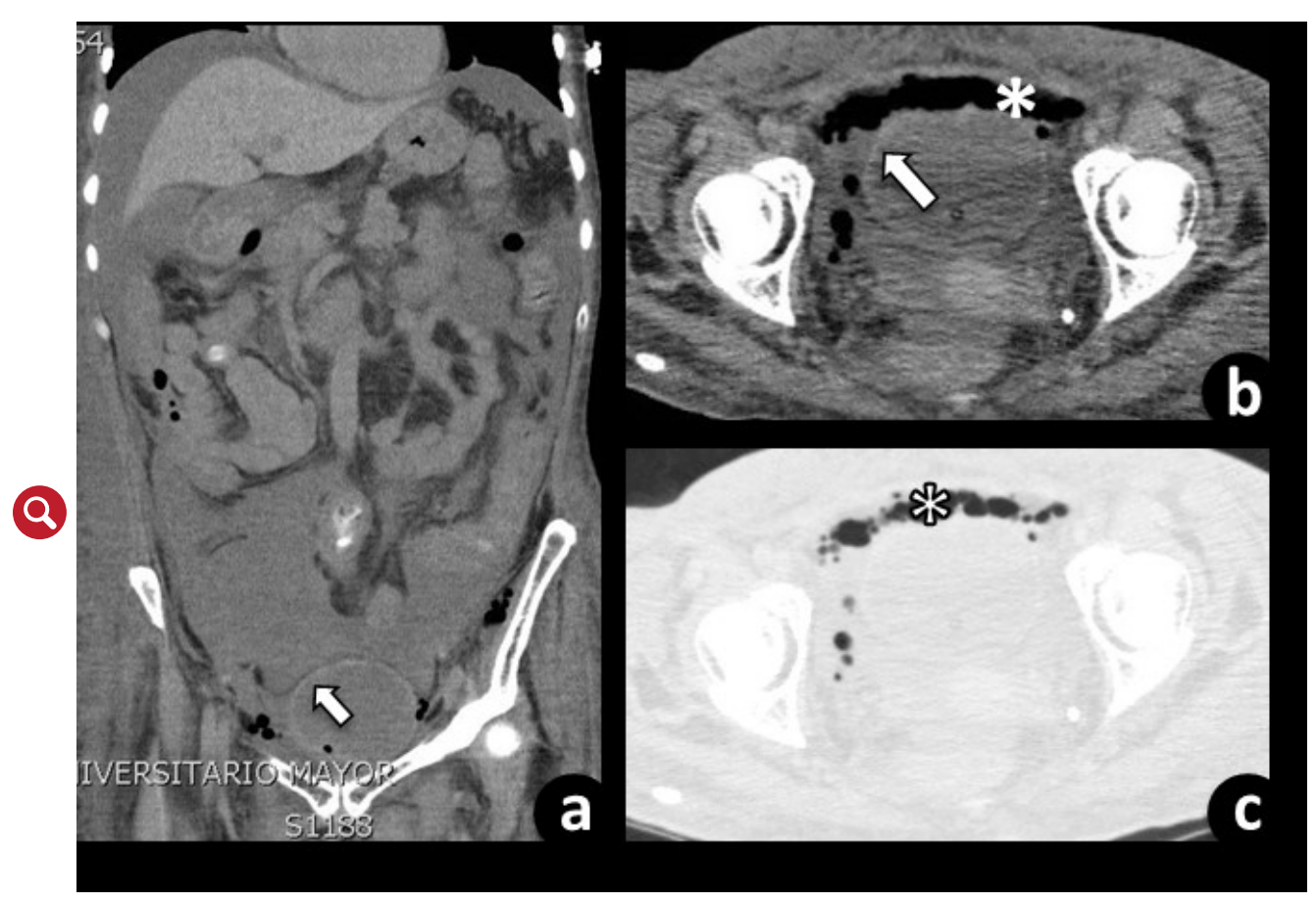

Figura 3. Tomografía abdominal contrastada, cortes axiales y reconstrucción coronal. En la imagen se evidencia ausencia de aire en la pared vesical (asterisco) (a) con diminución del aire en el espacio retroperitoneal (asterisco) (b y c) y aumento del líquido libre intraabdominal.

\section{Discusión}

La cistitis enfisematosa es la presencia de gas en la pared vesical, secundaria a colonización por microorganismos formadores de gas (3), mientras que el retroneumoperitoneo es la presencia de gas en el espacio retroperitoneal (4).

El primer caso descrito de cistitis enfisematosa se diagnosticó a partir de la manifestación clínica de neumaturia en 1671. En los años 80's del siglo pasado, Eisenlohr describe el primer caso en autopsia (1). Más adelante, en 1961 Bailey et al. realizan la primera serie clínica con 19 casos y le asignan el nombre de "cistitis enfisematosa" $(\underline{3}, \underline{5})$.

Se trata de una complicación rara de las infecciones del tracto urinario, por lo que se desconocen datos de su prevalencia e incidencia (4). La cistitis enfisematosa es más frecuente en el género femenino con una relación mujer hombre de 1,8:1, y un pico de incidencia entre la sexta y octava década de la vida $(\underline{3}, \underline{6})$. 
Los factores de riesgo descritos son: diabetes mellitus, vejiga neurogénica, cateterismo vesical, fístula vesicorectal, enfermedad renal terminal infección urinaria recurrente y obstrucción del tracto urinario.
Los factores de riesgo descritos son: diabetes mellitus (62,2 \%) (7), vejiga neurogénica, cateterismo vesical, fístula vesico-rectal, enfermedad renal terminal infección urinaria recurrente y obstrucción del tracto urinario (ㅇ). Se ha descrito en asociación a enfermedades como el lupus eritematoso sistémico o hematológicas $(\underline{7}, \underline{9}, \underline{10})$.

Existe un caso reportado en la literatura, como el presentado, en el que el único factor de riesgo identificado es la inmunosupresión farmacológica con esteroides (dosis diaria de prednisolona mayor de $10 \mathrm{mg}$ ) (11). En el contexto de lupus eritematoso sistémico sólo hay un caso reportado en la literatura de cistitis enfisematosa, cuyo factor predisponente identificado fue el antecedente de vejiga neurogénica por diabetes mellitus tipo 2 (12).

El mecanismo fisiopatológico de la cistitis enfisematosa incluye: infección por microrganismos anaerobios productores de gas, glucosuria que facilita la fermentación, disminución del flujo sanguíneo e inmunosupresión ()․

El tipo de fermentación de la glucosa en la orina depende del microrganismo colonizador: alcohólica o láctica (Streptococcus y Lactobacillus), de ácidos mixtos y butanediol (Enterobacter), de ácido propiónico (Propionibacterium) y de ácido butírico (Clostridium). En los pacientes no diabéticos la albumina, lactosa urinaria o las proteínas tisulares actúan como sustratos para la formación de gas (므, $\underline{6}, \underline{7})$.

La fermentación de la glucosa lleva a acumulación de ácidos los cuales disminuyen el pH local. Cuando el pH es menor de seis las enzimas producidas por los microrganismos disocian los ácidos y se produce gas compuesto por nitrógeno, hidrógeno, oxígeno y dióxido de carbono $\left(\mathrm{CO}_{2}\right)$, el cual se recolecta aumentando la presión de los tejidos con oclusión del flujo sanguíneo e isquemia $(\underline{1}, \underline{2}, \underline{6}, \underline{7}, \underline{13})$.

El déficit de flujo sanguíneo genera mal absorción del gas vesical que junto con la isquemia y el estado de inmunosupresión del paciente facilita la reproducción de los microrganismos y la perpetuación de la infección con mayor formación de gas. Este gas pasa por la mucosa de la vejiga hacia el espacio infraperitoneal alrededor de la base de la vejiga y posteriormente migra hacia el tejido prostático (en el caso de los hombres), periuretral, escroto, fosa isquiorectal y espacio retroperitoneal $(\underline{6}, \underline{7}, \underline{13})$.

En el 90 \% de los casos hay aislamiento microbiológico (1), siendo los microrganismos aislados con mayor frecuencia Escherichia coli (58-60 \%) y Klebsiella pneumoniae (10-21\%). Otros microrganismos aislados incluyen Enterobacter aerogenes (7 \%), Clostridium perfringens (6 \%), Proteus mirabilis, Citrobacter, Streptococcus spp, Candida albicans, Pseudomonas aeruginosa, Entercococcus faecalis, Staphylococcus aureus, Clostridium welchii, Candida tropicalis y Aspergilus fumigatus $(\underline{3}, \underline{6}, \underline{2}, \underline{10})$. Existen solo dos casos reportados en la literatura con extensión del aire al espacio retroperitoneal con aislamiento de Escherichia coli en el urocultivo (1,2).

Las causas del retroneumoperitoneo incluyen ruptura de víscera hueca secundaria a causas médicas (úlcera péptica, colitis, etc.), trauma abdominal, iatrogenia, infección o inflamación de órganos sólidos retroperitoneales como pancreatitis, pielonefritis enfisematosa, etc. (14).

Se describe un amplio espectro de manifestaciones clínicas, desde pacientes asintomáticos (7 \%) hasta estados de choque séptico, como la evolución del caso presentado (1). El síntoma más frecuente es dolor abdominal (80 \%) seguido por síntomas 
El síntoma más frecuente es dolor abdominal seguido por síntomas irritativos urinarios, dentro de los que la retención urinaria, hematuria y disuria son los más frecuentes. irritativos urinarios (53\%), dentro de los que la retención urinaria, hematuria y disuria son los más frecuentes $(1,7)$.

También se han descrito síntomas generales como nausea (60\%), fiebre (52,9\%) y diarrea (18,2 \%) (7). El síntoma más específico es la neumaturia, aunque es poco frecuente $(26,7 \%)(\underline{4}, \underline{6}, \underline{7})$. La presencia de fiebre es sugestiva de pielonefritis, sin embargo el 30-50 \% de los pacientes puede estar febril sin presentarla (4).

La cistitis enfisematosa se diagnostica a través de imágenes diagnósticas (radiografía simple de abdomen o tomografía computarizada de abdomen) o visualización directa a través de cistoscopia, laparotomía o autopsia (ㅁ).

Las alteraciones en la radiografía simple de abdomen incluyen un halo radiolúcido que delimita la pared vesical con o sin aire, en el área de proyección de la vejiga. La tomografía computarizada permite realizar el diagnóstico diferencial, logra una mayor caracterización de la distribución y extensión del aire. En ésta se identifican burbujas de aire en la pared vesical, el cual se puede extender al espacio perivesical, retroperitoneal, intraperitoneal y a tejido celular subcutáneo. Sólo se conoce de dos casos reportados con extensión del aire al espacio retroperitoneal (1,2).

En la serie de Grupper et al. ( $\underline{7})(n=53)$ la tomografía computarizada tuvo mayor rendimiento que la radiografía simple para diagnosticar cistitis enfisematosa (100\% vs $11,3 \%)$. Hallazgos similares fueron reportados en la serie de Kuo et al. (15) donde la radiografía simple de abdomen fue diagnóstica solo en el $13 \%$ de los pacientes. En esta serie se realizó ultrasonografía abdominal al 24,5\% de los pacientes, en la que solo el 46,1 \% presentó alteraciones, sin ser diagnósticas.

De acuerdo a los hallazgos imagenológicos el diagnóstico diferencial incluye fístula vesico-intestinal, vesico-vaginal e instrumentación del tracto urinario bajo $(\underline{2}, \underline{4}, \underline{8})$ (cuadro 1).

Cuadro 1. Diagnósticos diferenciales

\begin{tabular}{|c|c|c|c|}
\hline Diagnóstico & Ultrasonografía & Radiografía & Tomografía \\
\hline Cistitis enfisematosa & $\begin{array}{l}\text { Engrosamiento de } \\
\text { la pared de la vejiga, } \\
\text { áreas ecogénicas } \\
\text { perivesicales }(\underline{16})\end{array}$ & $\begin{array}{l}\text { Halo radiolúcido } \\
\text { delimitando la pared } \\
\text { vesical } \underline{(\underline{16})}\end{array}$ & $\begin{array}{l}\text { Aire en la pared vesical con o sin aire } \\
\text { en el lumen vesical. El aire se puede } \\
\text { extender al espacio perivesical, } \\
\text { retroperitoneal, peritoneal y tejido } \\
\text { celular subcutáneo (17) }\end{array}$ \\
\hline $\begin{array}{l}\text { Instrumentación de } \\
\text { tracto urinario bajo }\end{array}$ & Aire en el lumen vesical & $\begin{array}{l}\text { Aire en el lumen } \\
\text { vesical }\end{array}$ & $\begin{array}{l}\text { Aire en el lumen vesical con } \\
\text { engrosamiento de la pared vesical }\end{array}$ \\
\hline Fístula entero-vesical & $\begin{array}{l}\text { "Signo de pico" } \\
\text { ecogénico conectando } \\
\text { el lumen de un asa } \\
\text { peristáltica y la vejiga } \\
(\underline{18,19)}\end{array}$ & $\begin{array}{l}\text { Aire en el área de } \\
\text { proyección de la } \\
\text { vejiga }(\underline{18,19)}\end{array}$ & $\begin{array}{l}\text { Aire en el lumen vesical. En caso } \\
\text { de administración de medio de } \\
\text { contraste oral, presencia de este en } \\
\text { el lumen vesical. Engrosamiento de } \\
\text { la pared vesical adyacente a una asa } \\
\text { intestinal engrosada }(\underline{18,19)}\end{array}$ \\
\hline
\end{tabular}


El tratamiento incluye antibioticoterapia endovenosa de acuerdo a la sensibilidad del microrganismo, drenaje vesical continuo y control estricto de las cifras glicémicas. En la serie de Grupper et al. el grupo antibiótico más usado fue el de los betalactámicos con una media de duración de 10 días.
El tratamiento incluye antibioticoterapia endovenosa de acuerdo a la sensibilidad del microrganismo, drenaje vesical continuo y control estricto de las cifras glicémicas (16). En la serie de Grupper et al. (7) el grupo antibiótico más usado fue el de los betalactámicos $(60 \%)$ con una media de duración de 10 días.

Entre el 10-20 \% de los pacientes requieren manejo quirúrgico (cistectomía parcial o total, debridamiento quirúrgico) debido a la poca respuesta al manejo conservador, infecciones necrotizantes severas, signos de irritación peritoneal, neumoperitoneo o absceso perivesical $(\underline{1}, \underline{2}, \underline{4}, \underline{7})$.

Los dos casos reportados de retroneumoperitoneo fueron inicialmente manejados con antibióticos endovenosos y cateterización uretral, como en el caso descrito. Uno de éstos requirió manejo quirúrgico, debido al hallazgo de necrosis de la pared vesical en la tomografía de abdomen de control a los siete días (1,2).

La mortalidad reportada varía entre el 7 y el $20 \%(1,6)$. Cuando el gas migra a través

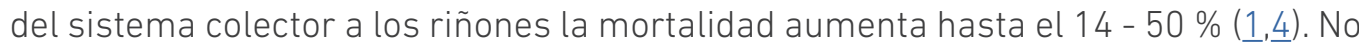
existe información sobre la mortalidad en los casos asociados con retroneumoperitoneo. De los dos casos reportados en la literatura, uno falleció a los cinco días de haber suspendido el antibiótico endovenoso, debido a la decisión familiar de limitar el esfuerzo terapéutico por las comorbilidades del paciente.

\section{Conclusión}

Entre las causas de retroneumoperitoneo debe considerarse cistitis enfisematosa con extensión extraperitoneal del aire desde la pared vesical.

\section{Bibliografía}

1. Sadek A-R, Blake H, Mehta A. Emphysematous cystitis with clinical subcutaneous emphysema. Int J Emerg Med. Springer Open Ltd; 2011;4(1):26. link

2. De Baets K, Baert J, Coene L, Claessens M, Hente R, Tailly G. Emphysematous cystitis: report of an atypical case. Case Rep Urol. 2011;2011:1-5. link

3. Thomas A a, Lane BR, Thomas AZ, Remer EM, Campbell SC, Shoskes D a. Emphysematous cystitis: a review of 135 cases. BJU Int. 2007;100(1):17-20. link

4. Amano M, Shimizu T. Emphysematous Cystitis: A Review of the Literature. Intern Med. 2014;53(2):79-82. link

5. Bos D, Patal P, Ditullio S. case report Emphysematous cystitis : An atypical multiorganism presentation. 2014;8(4):8-10. link

6. Mokabberi R, Ravakhah K. Emphysematous urinary tract infections: diagnosis, treatment and survival (case review series). Am J Med Sci. 2007;333(2):111-6. link

7. Grupper M, Kravtsov A, Potasman I. Emphysematous cystitis: illustrative case report and review of the literature. Medicine (Baltimore). 2007;86(1):47-53. link

8. Yoshida K, Murao K, Fukuda N, Tamura Y, Ishida T. Emphysematous cystitis with diabetic neurogenic bladder. Intern Med [Internet]. 2010;49(17):1879-83. Link 
9. Péres D, Blanco M, Lema J, Toucedo V, Novás S, Lamas P, et al. Cititis enfisematosa: A propósito de un caso. Arch Esp Urol. 2009;62(5):392-5. link

10. Ahsaini M, Kassogue A, Tazi MF, Zaougui A, Elammari JE, Khallouk A, et al. Emphysematous cystitis and emphysematous pyelitis: a clinically misleading association. Pan Afr Med J [Internet]. 2013;16:18. link

11. Yoshino T, Ohara S, Moriyama H. Case report emphysematous cystitis occurred in the case treated with steroid for autoimmune hepatitis. 2013;2013:3-6. link

12. Granel B, Serratrice J, Juhan V, Rey J, Lechevalier E, Disdier P, et al. Lupus cystitis: A possible additive risk factor for emphysematous cystitis in diabetes mellitus: discussion about one case. Lupus. 2000;9(1):72-3. link

13. Sereno M, Gómez-Raposo C, Gutiérrez-Gutiérrez G, López-Gómez M, Casado E. Severe emphysematous cystitis: Outcome after seven days of antibiotics. Mcgill J Med. 2011;13(1):13. link

14. Diaz N, Forero O, Ulloa L, Camargo C, Carrillo J, Rivera A. Gas extraluminal abdominal en imagen. Causas y significado clínico. Rev Colomb Radiol. 2008;19(2):2370-86. link

15. Kuo C-Y, Lin C-Y, Chen T-C, Lin W-R, Lu P-L, Tsai J-J, et al. Clinical features and prognostic factors of emphysematous urinary tract infection. J Microbiol Immunol Infect. 2009;42:393-400. link

16. O'Connor La, De Guzman J. Emphysematous cystitis: a radiographic diagnosis. Am J Emerg Med. 2001 May;19(3):211-3. link

17. Eken A, Alma E. case report Emphysematous cystitis : The role of CT imaging and appropriate treatment. 2013;7:11-3. link

18. Kelly J. Vesico-vaginal and recto-vaginal fistulae. J Obstet Gynaecol. 1998;18(3):249-51. Link

19. Golabek T, Szymanska A, Szopinski T, Bukowczan J, Furmanek M, Powroznik J, et al. Enterovesical fistulae: aetiology, imaging, and management. Gastroenterol Res Pract. 2013;2013(9). link 